\title{
\begin{tabular}{l|l} 
POLITIQUES \& & Politiques et management public
\end{tabular}

\section{L'exercice de la responsabilité managériale dans la sphère publique. Le cas des responsables de programme dans la LOLF}

The exercise of managerial responsibility in the public sector. The case of LOLF program managers

\section{Fatima Yatim}

\section{OpenEdition}

\section{Journals}

\section{Electronic version}

URL: http://journals.openedition.org/pmp/7165

ISSN: 2119-4831

Publisher

Institut de Management Public (IDPM)

\section{Printed version}

Date of publication: 15 June 2014

Number of pages: $319-336$

ISBN: 978-2-7430-2007-1

ISSN: 0758-1726

Electronic reference

Fatima Yatim, «L'exercice de la responsabilité managériale dans la sphère publique. Le cas des responsables de programme dans la LOLF », Politiques et management public [En ligne], Vol 31/3 | 2014, mis en ligne le 29 décembre 2017, consulté le 14 juin 2019. URL : http:// journals.openedition.org/pmp/7165 


\section{L'exercice de la responsabilité managériale dans la sphère publique. Le cas des responsables de programme dans la LOLF}

Fatima Yatim

EA Management des organisations de santé École des hautes études en santé publique

\section{Résumé}

En France, la loi Organique relative aux Lois de Finances (LOLF) fait évoluer la logique de l'action publique pour imposer une culture de gestion par les résultats. Ce faisant, elle implique de nombreux changements notamment en termes d'évolution du rôle des hauts fonctionnaires. Dans cet article, nous nous intéressons plus particulièrement aux responsables de programmes de la LOLF et nous nous interrogeons sur les marges de manœuvre dont ils disposent pour exercer la responsabilité managériale que la réforme leur attribue. Nous nous appuyons sur une recherche documentaire basée sur des rapports publics et en particulier le rapport bilan de la LOLF réalisé par la Cour des Comptes fin 2011. Nous mettons en évidence et expliquons l'insuffisance des marges de manœuvre des responsables de programme ainsi que la tension existante entre leur responsabilité managériale, celle des politiques et celles d'autres acteurs administratifs. Nous en discutons les implications en matière de partage des responsabilités et de reddition des comptes.

(c) 2014 IDMP/Lavoisier SAS. Tous droits réservés

Mots clés : LOLF, responsables de programme, responsabilité managériale, gestion par les résultats.

\section{Abstract}

The exercise of managerial responsibility in the public sector. The case of LOLF program managers. In France, the Constitutional Bylaw on Budget Acts (LOLF) has introduced a change in the logic of public policies towards a culture of results-based management. The reform entails many changes, notably regarding the role of Senior Officials. In this article, we study more specifically the case of LOLF program managers by questioning their leeway for the implementation of the managerial responsibilities assigned to them by the reform.

*Auteur correspondant : fatimayatim15@gmail.com

doi :10.3166/pmp.31.319-336 @ 2014 IDMP/Lavoisier SAS. Tous droits réservés 
For this research, we rely on a documentary analysis of public reports and more especially, on a report on LOLF published in 2011 by the French Cour des Comptes. We highlight and explain the program managers's limited leeway as well as the existing triple tension between their managerial responsibilities and those of political and administrative actors. We discuss the implications of this situation in terms of actors' respective responsibility and accountability. @ 2014 IDMP/ Lavoisier SAS. Tous droits réservés

Keywords: Constitutional Bylaw on Budget Acts (LOLF), program manager, managerial responsibility, results-based management.

\section{Introduction}

Suite à l'ordonnance de 1959, trois règles définissaient la gestion des finances publiques françaises : l'unité ${ }^{1}$, l'universalité ${ }^{2}$ et l'annualité 3 budgétaire (Bartoli, 2009). Ce cadre réglementaire qui se voulait égalitaire et juste présentait de nombreuses limites : déséquilibre des pouvoirs entre l'exécutif et les assemblées démocratiques, lourdeur et complexité du système comptable, multiplication des niveaux de décision et de contrôle, etc. (Waintrop et Chol, 2004; Dussart et Esplugas, 2000). Pour pallier ces lacunes, dont les effets paralysants ont été largement démontrés depuis les années 60 (Albertini, 2000), la Loi Organique relative aux Lois de Finances (dite LOLF), votée en août 2001 et mise en place en janvier 2006, constitue une évolution importante en innovant sur deux plans. Sur le plan politique, la LOLF participe de l'évolution des traits du système de pouvoir à travers le renforcement du rôle du Parlement dans l'élaboration et le suivi des lois de finances (Hochedez, 2004). Sur le plan économico-gestionnaire, elle met l'accent sur le fait que tout acteur public, État compris, doit démontrer son efficacité (Barilari et Bouvier, 2004).

Pour répondre à cette double finalité, la LOLF introduit une nouvelle dynamique centrée sur une logique de gestion par les résultats conformes aux principes du New Public Management (NPM) (Pollitt et Bouckaert, 2004). Ainsi, à l'instar de l'ensemble des réformes $N P M$ opérées dans d'autres pays, la LOLF fait évoluer le cadre global de l'action publique pour se centrer davantage sur les performances des organisations publiques en termes d'efficience et d'efficacité politique, économique et sociale (Mazouz et Mathais, 2004). Aussi, si les principes du NPM ont été beaucoup étudiés, peu de travaux ont porté sur les conditions organisationnelles et institutionnelles de leur mise en œuvre (Bezes, 2005 ; De Vesscher et Varone, 2004). Plus particulièrement, l'évolution du rôle et des missions des hauts fonctionnaires a été peu étudiée (Hood et Lodge, 2004). Ceci est également vrai pour le cas français dans la mesure où les travaux sur la réforme LOLF se sont davantage concentrés sur les principes mêmes de la gestion par les résultats et du NPM d'un point de

\footnotetext{
'Qui se traduit par la production d'un seul document à soumettre au Parlement pour proposer l'ensemble des dépenses et recettes de l'État.

${ }^{2}$ Admettant deux niveaux : une inscription budgétaire formelle de toutes les recettes et dépenses sans possibilité de contraction ni de compensation entre ces dernières, et une logique de transversalité de l'intérêt général selon laquelle toutes les recettes ne peuvent être affectées à une dépense donnée.

${ }^{3}$ Impliquant que la loi des finances prévoit et autorise pour chaque année l'ensemble des ressources et des dépenses de l'État suite au vote du parlement.
} 
vue technique et/ou politique (Emery et Giauques, 2005).

Dans cet article nous traitons du cas des hauts fonctionnaires, responsables de programme (RP), nommés par leurs ministres de rattachement pour assurer le pilotage de la mise en œuvre des politiques publiques et en rendre compte. Nous nous intéressons plus spécifiquement à l'exercice de leur responsabilité managériale et nous posons la question des marges de manœuvre dont ils disposent. Le matériau empirique est fondé sur une recherche documentaire mobilisant différents rapports publics et plus particulièrement le rapport bilan de la LOLF établi par la Cour des Comptes fin 2011. L'analyse effectuée nous conduit à mettre en évidence les facteurs limitatifs de l'exercice de la responsabilité managériale : chevauchement des périmètres d'action, de compétences et d'autorité; insuffisance des marges d'action pour l'animation des échelons inférieurs; et lacunes en matière d'incitations à rendre compte et à faire l'objet de sanctions. Nous montrons ainsi qu'en introduisant cette dimension intermédiaire, la dimension managériale, entre le politique et l'administratif sans réellement remettre en cause le modèle existant, la LOLF appelle une clarification des modes de partage des responsabilités et des dispositifs de reddition des comptes.

L'article est structuré en trois parties. Dans une première partie nous revenons sur les principes de la gestion par les résultats et sur la diversité des approches selon les pays et nous mettons l'accent sur les implications en matière de responsabilité managériale pour dégager une grille d'analyse. Dans une deuxième partie, nous explicitons plus en avant les spécificités de la réforme introduite par la LOLF ainsi que le rôle des responsables de programme et nous présentons les résultats de notre recherche documentaire. Dans la troisième partie, nous discutons les implications de la LOLF en tant que réforme de type NPM.

\section{Gestion par les résultats et responsabilité des managers publics}

Bien que les vocables employés diffèrent; gestion par les résultats, management par les résultats ou encore gestion ou management par la performance, la doctrine du NPM demeure similaire; il s'agit de mettre les résultats des organisations publiques au cœur de leur fonctionnement pour en évaluer la performance à différents niveaux. Évaluée d'un point de vue politique, social et économique, la performance englobe les résultats en termes de qualité des services rendus (efficacité), en termes de qualité de la gestion des dépenses publiques (efficience) et en termes de qualité des décisions prises (transparence des processus de décision) (Mazouz et Mathais, 2004). La gestion par les résultats obéit ainsi à un certain nombre de fondements et principes qui, sans être universels, présentent des points de convergence multiples et mobilisent des outils comparables; contrôle de gestion, indicateurs de mesure de la performance, évaluation, contractualisation, etc. Le but est d'orienter les organisations publiques vers une intégration accrue en diffusant des normes communes qui visent à relativiser les spécificités sectorielles, organisationnelles, locales, professionnelles (Eymeri, 2005). Cependant, sa mise en pratique dépend du contexte politique, sociétal et économique du pays l'accueillant.

\subsection{Une doctrine commune mais des approches diverses}

Les réformes menées dans les différents pays adoptent des approches diverses. Au Royaume-Uni la démarche est basée sur le modèle marchand procédant par comparaison 
public/privé (Pollitt et Bouckaert, 2004). La gestion par les résultats est articulée autour de la mise en concurrence des différents acteurs publics et/ou privés avec l'assouplissement des structures publiques. Le marché, ou du moins ses principes de fonctionnement, sont au centre de la gestion publique avec une pratique poussée de la délégation via des mouvements de privatisation et d'externalisation de secteurs entiers de l'action publique. Aux Pays-Bas, la gestion par les résultats est orientée selon un modèle de gouvernance par réseaux avec une séparation nette entre les niveaux opérationnel et stratégique (Le Galèse, 2003). Les décisions sont décentralisées afin de permettre de mieux répondre aux besoins de l'environnement en accordant aux acteurs publics une plus grande autonomie d'action et une plus grande flexibilité. En contrepartie, les responsables publics sont tenus d'assumer pleinement la responsabilité de leurs décisions et de leurs performances car l'évolution de leur carrière en dépend. Dans d'autres pays, le Canada à titre d'exemple, la gestion par les résultats est acceptée dans sa dimension organisationnelle intégrée avec une approche systémique et multidimensionnelle de la performance, où l'accent est davantage mis sur le principe d'autonomie et sur la simplification des processus que sur l'assouplissement des structures (Mazouz et Rochet, 2005). La notion d'engagement est cruciale, qu'il s'agisse d'engagements contractuels formels ou non, et qu'il s'agisse de partenaires internes ou externes au domaine public.

La première approche met ainsi l'accent sur l'évolution du statut et du rôle des entités publiques pour orienter leurs modes de fonctionnement, la seconde privilégie les mécanismes de coordination externe avec l'élargissement des parties prenantes autour de l'État, tandis que la dernière vise davantage l'optimisation des processus de l'action publique et l'engagement des acteurs. Plusieurs facteurs peuvent expliquer cette diversité (Eymeri, 2005); la structuration des coalitions entre acteurs réformateurs et les résultats des rapports de force en la matière (Parlement, haute administration, experts externes) (Bezes, 2005); la nature du régime et du jeu politique entre Parlement, Premier-Ministre et Gouvernement qui définit les règles de répartition du pouvoir (Bevir et al., 2003); le contexte social (coalitions entre acteurs sociaux, spécificités et poids des identités professionnelles, etc.) ainsi que le contexte économique (contexte budgétaire, spécificités sectorielles, etc.), qui peuvent tous deux légitimer ou non les réformes et en orienter le périmètre et la profondeur (Rochet, 2005).

Il existe toutefois, un autre facteur fondamental qui a une influence déterminante sur le plan organisationnel et institutionnel; à savoir la structuration socio-historique des bureaucraties et la nature des relations qui lient ces dernières au politique (James, 2001). En effet, quelle que soit l'approche choisie, dans l'ensemble des pays adoptant la gestion par les résultats il est avant tout question de guider le processus d'allocation des ressources afin d'orienter la prise de décision publique (Rochet, 2005). Or, comme le soulignent Mazouz et Mathais (2004), la budgétisation est l'un des éléments les plus politiques dans le processus de décision publique. La gestion par les résultats amène en effet à poser la question du partage des responsabilités, au sein des administrations qui s'engagent sur les résultats, et dans les rapports que ces dernières entretiennent avec les politiques, qui eux définissent les objectifs et les moyens.

\subsection{Relations politico-administratives et partage des responsabilités}

Sur le plan politique, la responsabilité implique le devoir de rendre compte de ses 
actions et d'en assumer les conséquences devant le Parlement et autres assemblées représentatives (Eraly et Hindriks, 2007). Toutefois, l'exercice de cette responsabilité implique aussi que l'autorité politique, légitimement acquise par la représentativité, soit déléguée du ministre vers l'administration selon une logique hiérarchique pyramidale (Mattei, 2007). La délégation doit ainsi couvrir les ressources ainsi que la mise en œuvre des actions en contrepartie d'un contrôle politique ex ante (Verhoest et al., 2004). Le ministre demeure néanmoins responsable du pilotage de l'appareil administratif, conformément aux règles du modèle weberien en matière de structuration des relations entre pouvoir politique et administration (Mattei, 2007).

Par rapport à ce schéma de partage des responsabilités, la gestion par les résultats introduit une remise en question importante en conditionnant l'efficacité à une plus grande autonomie des administrations et des gestionnaires publics (Pollitt, 2002). Sur le plan organisationnel, elle implique donc une évolution des relations entre les responsables publics pour passer d'une forme hiérarchique verticale à une forme plus horizontale car la logique des résultats impose une vision processuelle transversale (Hood et Lodge, 2004). Sur le plan institutionnel, elle implique l'évolution du contrôle politique de l'appareil bureaucratique pour passer d'un contrôle a priori à un contrôle a posteriori dans la mesure où l'accent est mis sur les résultats et non sur les moyens (De Vesscher et Varone, 2004).

La gestion par les résultats fait donc émerger une autre logique de responsabilité, la responsabilité managériale, où l'imputabilité est ex post et est fondée sur les performances et non sur la conformité de la mise en œuvre (Mattei, 2007). Cette nouvelle forme de responsabilité est alors interne et s'appuie sur une autre autorité légitime qui vient du processus de gestion et de sa maîtrise (Mulgan, 2000). Toutefois, pour être effective, la responsabilité managériale suppose un partage des pouvoirs sur les ressources mais également dans le processus de prise de décision. En effet, trois conditions sont identifiées dans la littérature en matière d'exercice de la responsabilité managériale par les hauts fonctionnaires (De Visscher, 2004) : une implication dans les processus de prise de décision; des marges d'action pour la gestion des ressources; et une pratique de la reddition des comptes suivie de sanctions positives ou négatives. Or, la sphère publique présente de nombreuses particularités : spécificités des statuts juridiques des entités et des personnels, uniformité de la règle administrative et prééminence de la hiérarchie, distance entre les lieux où se prennent les décisions et les lieux où elles s'appliquent, double direction politique et administrative des entités, etc. (Emery et Giauques, 2005).

La problématique qui se pose alors est celle de l'évolution du périmètre des responsabilités car la gestion par les résultats fait évoluer les relations qui lient l'ensemble des acteurs publics ; politiques et administratifs. En France, dans le cadre de la LOLF, cette problématique se pose de manière aiguë pour le cas des responsables de programme. Parce qu'ils doivent rendre compte des résultats des programmes devant leur ministre de rattachement et devant le Parlement, les responsables de programme sont en effet porteurs d'une responsabilité managériale qui les engage et engage leurs choix gestionnaires.

\subsection{Structuration des responsabilités dans la LOLF}

Contrairement à l'ordonnance de 1959 qui structurait les finances publiques par 
chapitres, la LOLF privilégie la logique des politiques publiques qu'elle structure en une série de programmes à l'intérieur des principales missions de l'État ${ }^{4}$. Le terme de programme ${ }^{5}$ 'est pas nouveau puisqu'il remonte à la réforme de la Rationalisation des Choix Budgétaires (RCB), mais la nouveauté réside dans le lien établi entre les objectifs de ces programmes, leurs résultats et le processus budgétaire. La nouvelle approche place la culture des résultats au centre de la démarche budgétaire en mettant l'accent sur l'évaluation et la reddition des comptes. Le schéma budgétaire de la LOLF fait alors apparaître différents niveaux et interactions.

En amont du processus figurent les grandes orientations en matière de politiques publiques définies par le Gouvernement et dont la charge revient aux ministres. Les activités aval d'évaluation et de contrôle sont-elles confiées à des instances transversales et plus particulièrement à la Cour des Comptes qui assure le contrôle financier, comptable et réglementaire mais évalue également la fiabilité et la qualité des bilans annuels en termes de résultats. Le Parlement quant à lui intervient au début et à la fin du processus budgétaire. En amont, il se prononce sur les Projets Annuels de Performance (PAP) et vote les autorisations de dépenses ${ }^{6}$. En aval, il intervient pour s'exprimer sur la tenue des engagements pris à l'aune des Rapports Annuels de Performance (RAP) et vote les autorisations de règlements.

En assurant le pilotage des activités intervenant entre ces phases amont et aval, les responsables de programme (RP) assument la responsabilité des résultats et doivent en rendre compte ${ }^{7}$. Ils sont ainsi en charge de l'animation du dialogue de gestion avec les échelons inférieurs : responsables de Budgets Opérationnels de Programme (BOP) et responsables des Unités Opérationnelles (UO). Comme nous le présentons de manière simplifiée dans la figure 1 ci-dessous, ils sont donc porteurs d'une responsabilité managériale qui doit s'exercer au sein d'un dispositif d'ensemble où il existe de nombreux acteurs aux périmètres d'action, d'autorité et de compétence enchevêtrés.

\footnotetext{
${ }^{4}$ II existe ainsi 33 missions ministérielles et interministérielles, réparties en 143 programmes ministériels (chiffres de 2010).

${ }^{5}$ Selon l'article 7 de la LOLF, le programme doit regrouper «les crédits destinés à mettre en œeuvre une action ou un ensemble cohérent d'actions relevant d'un même ministère et auquel sont associés des objectifs précis, définis en fonction de finalités d'intérêt général, ainsi que des résultats attendus et faisant l'objet d'une évaluation ».

6 Selon la pertinence des priorités définies, adéquation des moyens consacrés, soutenabilité des engagements pris.

${ }^{7}$ Le texte de la loi organique de 2001 ne précisait que de manière indirecte les prérogatives des responsables de programmes. Ce n'est qu'à l'occasion de la refonte du règlement général de la comptabilité publique que des précisions ont été apportées quant au statut et aux missions des responsables de programme : «pour chaque programme, un responsable est désigné par le ministre à la disposition duquel les crédits de programme ont été mis. Le responsable de programme établit le projet annuel de performances (...). II présente dans ce document les orientations stratégiques et les objectifs du programme et justifie les crédits et les autorisations d'emplois demandés. Il définit le périmètre des budgets opérationnels de programme et des unités opérationnelles et en désigne les responsables 》 (décret du 7 novembre 2012 relatif à la gestion budgétaire et comptable publique).
} 
Figure 1 : «Architecture et acteurs de la LOLF »

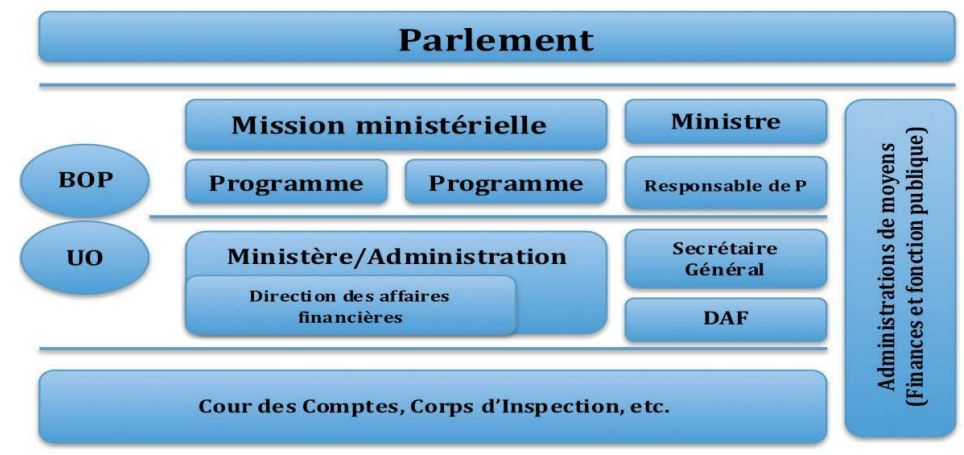

\section{Responsable de programme, une responsabilité managériale sous tension}

Du fait de leur positionnement, les RP sont au centre de la démarche de gestion par les résultats instaurée par la LOLF. Toutefois, comme pour beaucoup d'autres objectifs attendus de la réforme, leur rôle et la responsabilité managériale qui leur a été confiée ne semblent pouvoir s'affirmer. Dans cette deuxième partie nous nous intéressons plus spécifiquement aux marges de manœuvre dont disposent ces acteurs clés. Nous mettons ainsi l'accent sur trois principaux facteurs limitatifs de leur exercice de la responsabilité managériale : la faible implication dans le processus de prise de décision budgétaire en raison du chevauchement des périmètres d'action, de compétences et d'autorité ; l'insuffisance des marges de manœuvre pour la gestion des ressources au niveau des échelons inférieurs; et les lacunes en matière d'incitations à rendre compte et à faire l'objet de sanctions.

\section{Précisions méthodologiques}

Notre matériau de recherche est basé sur une recherche documentaire. Les sources privilégiées ont été des documents et rapports publics qui restituent les résultats des travaux préparatoires à l'élaboration de la réforme LOLF et ceux concernant les différentes étapes et dimensions de sa mise en œuvre. Parmi ces documents, le rapport bilan global de la réforme réalisé en 2011 par la Cour des Comptes nous a servis de source documentaire principale, enrichie par d'autres rapports de la Cour des Comptes et par des rapports parlementaires. Ce choix s'explique par le fait qu'il s'agit d'un rapport qui établit le bilan de cinq années de mise en œuvre et qui traite de l'ensemble des dimensions de la réforme sur le plan budgétaire, financier et comptable, mais également sur le plan politique et organisationnel. Il inclut ainsi une analyse détaillée et étayée par des faits concernant les difficultés et les avancées de la réforme. Il convient néanmoins de préciser que, parmi les données figurant dans le rapport, seules ont été analysées les données relatives à la question de la responsabilité et au rôle des RP. Le travail d'analyse a été entrepris selon une démarche progressive, procédant par itération entre sélection d'informations, exemples et faits pertinents pour répondre à la question de recherche, et interprétation selon le cadre conceptuel mobilisé (Wacheux, 1996). 
Ont ainsi été identifiés comme éléments pertinents, les faits, informations et exemples permettant d'éclairer les trois points suivant : 1) le rôle des RP dans les processus de prise de décision budgétaire ; 2) les marges d'action dont ils disposent pour la gestion des ressources financières et humaines ; 3 ) leurs capacités à rendre compte et à faire l'objet de sanctions. À ces trois points nous avons fait correspondre trois variables d'analyse. Le rôle en matière de décision a été analysé en s'intéressant aux périmètres d'actions des différents acteurs intervenant dans le processus de décision budgétaire. Les marges d'action pour la gestion des ressources ont été analysées selon la capacité des RP à animer les services opérationnels centraux et déconcentrés dépendant de leur autorité. Les capacités à rendre compte et à faire l'objet de sanctions ont été analysées au regard de l'existence d'incitations et de dispositifs reliant les performances des programmes au traitement individuel des RP. Il convient toutefois, de souligner que notre recherche documentaire comporte des limites en raison de la mobilisation de données indirectes comme seule source. Cependant, les données analysées présentent un degré de fiabilité acceptable car elles fournissent des informations, exemples et constats précis et en quantité significative pour permettre de répondre à la question de recherche et d'appréhender la problématique traitée.

\subsection{Le processus de prise de décision budgétaire}

Les RP doivent assurer le lien entre le politique qui définit les objectifs, l'administration qui les met en œuvre, et piloter cette mise en œuvre pendant l'année de l'exercice budgétaire. Dans les faits et en raison du chevauchement des périmètres d'action, de compétences et d'autorité, le pouvoir de décision des RP est fonction des équilibres établis avec trois autres acteurs majeurs de la LOLF que sont les ministres, les secrétaires généraux des ministères (SG) et les directeurs des affaires financières (DAF).

Sur le plan politique, les responsabilités des ministres couvrent largement celles des RP en raison de l'étendue du périmètre d'action des premiers. Le ministre dispose en effet à la fois de l'autorité politique, de l'autorité administrative en tant que chef des services, et de l'autorité financière en sa qualité d'ordonnateur principal de l'État. Ministre et RP partagent ainsi la responsabilité de prescription de l'exécution des dépenses et des recettes, et donc le pouvoir discrétionnaire de décision en matière de choix des dépenses. Toutefois, le pouvoir du ministre couvre également les choix de dépenses pour l'ensemble de la mission ministérielle, avec des possibilités de réaffectation des dépenses entre programmes en cours d'exercice budgétaire. Or, seule le RP est justiciable devant la Cour de discipline budgétaire et financière. Les rapporteurs de la Cour des Comptes explicitent ainsi les conséquences de l'ambiguïté du statut financier des ministres : « la responsabilité financière juridiquement impartie aux ministres s'avère donc peu effective au niveau des missions et tend à effacer celle des responsables de programme, qui ne sont pas "ès qualités ordonnateurs (...), supérieurs hiérarchiques de leurs collaborateurs (...), attributaires d'autorisations d'emplois". Les limites actuelles de l'exercice de la responsabilité financière des ministres, qui empêche une mise en jeu réelle des responsabilités de missions et programmes, appellent en conséquence à une clarification du statut financier des ministres ou à une clarification de l'imputabilité des décisions des uns et des autres dans une 'charte de responsabilité", des différentes autorités ». (p. 75-76). 
Sur le plan administratif, le périmètre d'action et de compétence du RP se chevauche avec les fonctions de SG qui dispose de l'autorité administrative au sein du ministère ${ }^{8}$. En outre, le SG peut également avoir le statut de prescripteur des dépenses et recettes, au niveau des programmes mais également au niveau de la mission ministérielle. D'autant que son pouvoir a été élargi dans le cadre de la RGPP (Révision Générale des Politiques Publiques) car c'est aux SG que revient la charge de la mutualisation des fonctions supports et donc les choix de dépenses en la matière. Ce dernier point est largement souligné dans le rapport de la Cour des Comptes qui précise : "la définition des mesures d'économies décidées dans le cadre de la RGPP a assez largement ignoré les responsables de programme es qualité. Ceux-ci apparaissent pour l'essentiel comme des exécutants de décisions prises dans un autre cadre. Le conseil de modernisation des politiques publiques a en effet choisi de confier le pilotage des mesures ministérielles aux secrétaires généraux, (...), la Cour souligne que les économies issues de la RGPP reproduisent une logique de gestion par nature de dépense (masse salariale et crédits de fonctionnement), dite par « titre », là où la LOLF engageait les gestionnaires à développer une vision large de leurs périmètres de crédits, constitués par des politiques publiques cohérentes, et ainsi dotés d'une exigence de résultats ». (p. 119).

Sur le plan financier, les fonctions de RP se chevauchent avec celles du DAF d'administration. Ce dernier doit assurer la coordination de la procédure de préparation budgétaire en lien avec les ministres et les RP, mais dispose dans les faits de prérogatives plus larges en raison de la proximité structurelle et relationnelle entre les DAF ministérielles et le ministère des finances. Le DAF est en effet, garant du respect du cadre réglementaire et des contraintes financières, il bénéficie du statut d'ordonnateur, et est de ce fait un acteur clé dans le processus de décision budgétaire. Le rapport de la Cour des Comptes souligne ainsi la diversité des relations de collaboration entre $\mathrm{RP}$ et DAF selon les ministères : « si les responsables des programmes « travail », « emploi » ou « gestion fiscale et financière de l'État et du secteur public local » négocient directement leurs crédits avec la direction du budget, ceux du ministère de la défense sont absents de la discussion menée par le seul directeur des affaires financières ». (p. 79)

En matière de décision budgétaire, le pouvoir des RP se trouve donc contraint par trois types d'autorité légitimes : l'autorité politique (du ministre), l'autorité administrative (du SG) et l'autorité financière (du DAF). Le chevauchement des périmètres d'autorité, mais également de compétences et de pouvoir de décision traduit finalement l'absence d'une revue générale des modes d'organisation de l'État. Le rapport de la Cour des Comptes souligne ainsi « entre le souci légitime de disposer de la vision la plus exhaustive possible des politiques publiques, la volonté incontestable, sur un plan démocratique, de valoriser certains choix politiques, celle, réaliste, du ministère chargé du budget et des comptes publics de disposer de structures budgétaires cohérentes, dotées d'une certaine taille critique, et celle des gestionnaires visant à maintenir une concordance la plus étroite possible entre leur périmètre de gestion et leur périmètre administratif, des compromis multiples, cumulant les défauts, ont été élaborés. » (p. 70). Ceci limite par conséquent « une mise en jeu réelle des responsabilités » (p. 72), comme précisé dans le rapport de la Cour des Comptes, et rend difficile l'imputabilité des

\footnotetext{
${ }^{8}$ La Fonction SG a été généralisée à partir de 2008, pour reprendre les attributions des anciennes directions d'administrations générales afin d'éviter l'éclatement des politiques publiques entre différentes directions au sein d'un même ministère. Le périmètre d'action et de compétence du SG couvre l'ensemble des directions en charge des fonctions supports, dont deux directions importantes : direction des affaires financières et direction des ressources humaines.
} 
décisions en matière de dépenses et la responsabilité financière qui en découle.

\subsection{Les marges de manœuvre gestionnaires}

Dans le cadre de la LOLF, de nombreuses marges de manœuvre ont été prévues au profit des RP notamment en termes d'allégement du contrôle financier et du fléchage des crédits. D'autres améliorations ont visé plus spécifiquement la gestion des ressources humaines via l'inclusion de la masse salariale dans le budget global et la simplification de la structure des corps. Toutefois, l'innovation majeure de la LOLF consistait en la mise en place du principe de la fongibilité asymétrique ${ }^{9}$ qui devait permettre aux RP de disposer de marges suffisantes pour gérer les services opérationnels centraux ou déconcentrés. Or, le bilan dressé dans le rapport de la Cour des Comptes sur la période 2006-2009 (p. 115) en montre le quasi-abandon comme l'indiquent les données du tableau 1 ci-dessous ${ }^{10}$.

Tableau 1 : Évolution de la fongibilité asymétrique entre 2006 et 2009 (en M€)

\begin{tabular}{|l|l|l|l|l|}
\hline & 2006 & 2007 & 2008 & 2009 \\
\hline Montant de la fongabilité assymétrique & 389 & 302 & 100 & 185 \\
\hline Dont fongabilité technique & 389 & 225 & 60 & 166 \\
\hline Dont fongabilité technique & & 77 & 40 & 19 \\
\hline
\end{tabular}

Plusieurs raisons expliquent ce constat. Tout d'abord, la nature même de la fongibilité, son caractère asymétrique, en limite la portée. En effet, la rigidité qu'elle impose pour la gestion de la masse salariale vient renforcer les contraintes existantes en matière de gestion des ressources humaines. Le rapport de la Cour des Comptes mentionne à titre d'exemple le cas du ministère des affaires étrangères où la masse salariale des expatriés est encore gérée par l'administration centrale et non par les ambassades tel que l'implique la LOLF. D'autre part, le contexte budgétaire a motivé de nouveaux contrôles et visas préalables entraînant par là même des lourdeurs supplémentaires. Les rapporteurs de la Cour des Comptes mentionnent ainsi : «des garde-fous puissants ont été posés pour limiter cette pratique : le décret du 27 janvier 2005 prévoit ainsi un avis préalable du contrôle financier sur tout mouvement de fongibilité asymétrique. Les circulaires successives du ministère du budget ont prescrit en outre un accord préalable du responsable de programme ou du directeur des affaires financières. Cette autorisation préalable est reprise dans de nombreuses chartes de gestion, par lesquelles les directions des affaires financières se voient confier un pouvoir d'instruction et de décision dans ce domaine ». (p. 115). Le rapport fait ainsi état d'une augmentation du taux de fléchage des crédits pour le ministère de l'intérieur à titre d'exemple, passant de $84 \%$ à 94,5\% entre 2007 et 2009. (p. 118).

\footnotetext{
${ }^{9}$ La fongibilité renvoie à la possibilité de redéployer les économies réalisées entre différents postes de dépenses dans la limite des enveloppes budgétaires autorisées. Elle est asymétrique dans la mesure où les économies réalisées sur des dépenses de fonctionnement ou d'investissement ne peuvent être redéployées en dépenses de masse salariale, seul l'inverse est possible.

${ }^{10}$ La fongibilité asymétrique technique correspond aux économies réalisées sur des dépenses de personnel et affectées à d'autres dépenses.
} 
Cette absence de marges de manœuvre se répercute essentiellement en termes de capacités des RP à animer la gestion des échelons inférieurs : responsables de budgets opérationnels de programme et d'unités opérationnelles. En outre, leurs capacités à déléguer se trouvent également réduites comme le précise le rapport de la Cour des Comptes : «les réticences à déléguer des capacités d'initiative au niveau local s'expliquent en partie par le sentiment des responsables de programme de ne pas disposer eux-mêmes de prise suffisante sur la gestion de leurs moyens. Le niveau des dépenses obligatoires limite leurs marges de manœuvre, sans que ce constat soit en aucune façon lié à la mise en auvre de la LOLF». (p. 120).

Cette incapacité est par ailleurs renforcée par des difficultés organisationnelles. En effet, les BOP et UO traduisent dans les faits l'héritage des structures administratives verticales et non pas une logique de déclinaison descendante telle que l'implique la LOLF. De nombreux cas sont ainsi cités dans le rapport de la Cour des Comptes «dans son référé de mai 2008 relatif aux pouvoirs des préfets, à l'organisation, et à l'action des services de l'État dans les régions et départements, la Cour avait jugé que «les responsables se multiplient, mais les responsabilités se diluent; la territorialisation s'accrô̂t, mais par juxtaposition de maillons de chaînes verticales ». (p. 65); « ces différences s'expliquent en partie par les décalages plus ou moins marqués entre les unités de gestion budgétaires (programmes, budgets opérationnels de programme et unités opérationnelles) et les organisations. En effet, au ministère de la défense, chaque responsable au niveau déconcentré ne gère qu'un seul budget opérationnel de programme et n'a donc qu'un dialogue unique à mener avec son responsable de programme. Dans le cas des directions régionales de l'environnement, de l'aménagement et du logement par exemple, les directeurs régionaux sont les interlocuteurs de quatre responsables de programme, limitant de fait la possibilité d'articuler chaque dialogue autour d'un nombre important de réunions. »(p. 102).

\subsection{Reddition des comptes et sanctions}

Selon les textes de la réforme, les RP présentent et justifient les résultats atteints devant leur ministre de rattachement mais également devant le Parlement ${ }^{11}$. Ceci suppose donc qu'il existe une certaine cohérence entre les critères utilisés par le Parlement, qui évalue l'atteinte des objectifs des politiques publiques, et ceux utilisés par le ministre, qui évalue l'atteinte des objectifs des crédits délégués dans le cadre du programme. Or, comme le souligne le rapport de la Cour des Comptes, les lettres de mission des RP témoignent : « du manque de cohérence entre objectifs et indicateurs fixés par les ministres et objectifs présentés au Parlement dans le cadre des Projets Annuels de Performances. (...) Cette absence d'articulation conduit dans les faits les responsables à privilégier les objectifs et les cibles définis par leur supérieur hiérarchique, le ministre, dans leurs lettres de mission, plutôt que ceux présentés au Parlement dans les documents budgétaires » (p. 162).

Les raisons de ce manque de cohérence remontent à l'élaboration de la nomenclature LOLF, lorsque le Gouvernement a opté pour une démarche ascendante, contrairement aux avis des parlementaires qui préconisaient, pour éviter la reproduction des organigrammes ministériels, que les missions soient définies selon les politiques publiques et déclinées par programmes et actions. Or, malgré de nombreux aménagements, la logique reste davantage

\footnotetext{
${ }^{11}$ Le choix de confier la responsabilité de la présentation des résultats devant le Parlement aux RP s'explique
} par le fait que l'évaluation est opérée au niveau des programmes et non pas au niveau des missions. 
orientée selon les périmètres ministériels et administratifs et non selon les objectifs des politiques publiques. Le rapport de la Cour des Comptes cite ainsi l'exemple du programme de l'enseignement du second degré public de la mission «enseignement scolaire » qui regroupe les moyens alloués aux collègues et aux lycées alors que l'objectif politique du socle commun des compétences et des connaissances implique que les moyens des collègues soient regroupés dans le même programme que ceux de l'école primaire (p. 101).

L'insuffisance des incitations pour rendre compte de l'atteinte des objectifs des programmes par les RP est renforcée par ailleurs par un certain désintérêt de la part des parlementaires eux-mêmes. De nombreuses raisons sont évoquées dans le rapport de la Cour des comptes : abondance de l'information et documents à analyser ${ }^{12}$; calendrier budgétaire inadapté ; abondance des indicateurs et leur faible durée de vie; et de manière générale un moindre intérêt pour les questions de performance par rapport aux questions de moyens. Ceci est mentionné à plusieurs reprises dans le rapport de la Cour des Comptes : "L'analyse des auditions réalisées depuis 2006 par le Parlement ainsi que l'enquête réalisée par la Cour auprès de l'ensemble des responsables de programme présente une situation contrastée : les auditions interviennent paradoxalement davantage en amont de l'exécution budgétaire, au moment de l'examen du projet de loi de finances, qu'a posteriori au moment de l'examen des résultats présentés dans le projet de loi de règlement. Par ailleurs, les auditions devant les commissions sont rares; elles sont plutôt pratiquées par les rapporteurs spéciaux, c'està-dire par chaque membre de la commission des finances chargé d'examiner les crédits relevant de tel ou tel ministre ». (p.81-82).

En outre, l'analyse de l'évolution du temps consacré à l'examen des projets de loi de finances initiales (PLF : objectifs/moyens) et celui consacré aux projets de loi de règlement (PLR : moyens/résultats), devant le Parlement, comme présentée ci-dessous dans le tableau 2, montre la constance d'un déséquilibre considérable alors que la LOLF devait augmenter le temps du débat autour des résultats (p. 144) :

Tableau 2 : Temps de discussion PLF/PLR

\begin{tabular}{|l|c|c|c|c|}
\cline { 2 - 5 } \multicolumn{1}{c|}{} & \multicolumn{2}{c|}{2004} & \multicolumn{2}{c|}{2009} \\
\cline { 2 - 5 } \multicolumn{1}{c|}{} & $\begin{array}{c}\text { Projet de loi de } \\
\text { finances initiale }\end{array}$ & $\begin{array}{c}\text { Projet de loi de } \\
\text { Règlement }\end{array}$ & $\begin{array}{c}\text { Projet de loi de } \\
\text { finances initiale }\end{array}$ & $\begin{array}{c}\text { Projet de loi } \\
\text { de Règlement }\end{array}$ \\
\hline $\begin{array}{l}\text { Assemblée } \\
\text { nationale }\end{array}$ & $160 \mathrm{~h} 28$ & $2 \mathrm{~h} 25$ & $108 \mathrm{~h} 32$ & $3 \mathrm{~h} 30$ \\
\hline Sénat & $138 \mathrm{~h} 55$ & $7 \mathrm{~h} 05$ & $134 \mathrm{~h} 25$ & $3 \mathrm{~h} 45$ \\
\hline
\end{tabular}

Vis-à-vis du Parlement, il n'existe donc pas pour les RP d'incitations à rendre compte, d'autant que le Parlement ne dispose d'aucun pouvoir de sanction. La loi ne prévoit pas en effet de dispositifs et n'accorde aucune autorité au Parlement en la matière. Seul le ministre est responsable, mais essentiellement devant le Premier-Ministre et devant le

\footnotetext{
12 Le nombre de pages des Projets Annuels de Performances, annexés au projet de loi de finances, a ainsi évolué de $42 \%$ passant de 5260 en 2006 à 7489 en 2011 (p. 31). Toutefois, comme précisé dans le rapport, cette abondance d'information n'est pas synonyme d'efficacité : « sur un plan qualitatif, l'appréciation des parlementaires sur l'information qui leur est transmise est plus difficile à mesurer, même si celle-ci apparaît globalement plus lisible et compréhensible. (...) Rien n'indique cependant aujourd'hui que ces projets et rapports annuels de performances satisfont complètement au besoin d'information du Parlement ». (p. 31)
} 
Président de la République. Ainsi le soulignent les rapporteurs de la Cour des Comptes : « de manière concrète, l'audition des responsables de programme n'a aucune conséquence directe sur eux puisque le Parlement n'a aucune autorité sur le responsable de programme. Celui-ci ne peut être sanctionné que par le ministre auquel il est rattaché. L'audition du responsable de programme sert donc à éclairer le Parlement sur la responsabilité politique du ministre » (p.81). En outre, cette lacune en matière d'incitations à rendre compte et de dispositifs de sanction, positive ou négative, est également vraie dans le lien de subordination qui lie le ministre au RP. Il n'existe en effet pas de dispositifs de sanction en lien direct avec les performances réalisées dans le cadre des programmes. Le décret du 21 avril 2006 qui instaure une rémunération à la performance s'adresse aux directeurs d'administration et ne cible pas les fonctions de RP, bien que les deux fonctions se confondent souvent. Le rapport de la Cour des Comptes pointe ainsi les limites de ce dispositif et souligne : « une implication inégale selon les ministères, avec une forte hétérogénéité dans les lettres des missions des ministres, lorsqu'elles existent, en termes de formalisation, de précision et d'objectivation des résultats à atteindre ». (p. 180). D'autant qu'il n'existe pas de consensus sur la logique de l'évaluation en ellemême : "la difficulté à trancher entre une approche visant à sanctionner la réalisation d'objectifs prédéfinis et une autre, consistant à apprécier de manière plus individuelle les compétences managériales du cadre dirigeant. Il n'existe pas formellement de répartition entre ces deux volets de l'appréciation de la performance ». (p. 180).

Plusieurs facteurs convergent ainsi pour complexifier l'exercice de la responsabilité managériale par les RP et réduire leur marge de manœuvre : la multiplication des niveaux de décision en termes de choix des dépenses à engager (ministres, SG, DAF); l'insuffisance des marges de manœuvre gestionnaires pour animer les services opérationnels centraux ou déconcentrés; le rôle limité du Parlement en matière d'évaluation des résultats et l'insuffisance des dispositifs de sanctions positives ou négatives, voire leur absence. En outre, les conséquences de ces facteurs ont été amplifiées par le contexte économique difficile qui a accompagné la mise en œuvre de la réforme. Les difficultés budgétaires et financières de l'État ont en effet compromis les promesses faites en termes d'assouplissement en contrepartie de l'exigence de la responsabilité et de reddition des comptes.

\section{Le NPM, réforme de la gestion publique et réforme de l'État}

Les difficultés rencontrées par les responsables de programme reflètent la tension qui existe entre la responsabilité managériale qui leur est attribuée, la responsabilité politique assumée par les ministres, et la responsabilité administrative et hiérarchique revenant à d'autres acteurs tels que les secrétaires généraux d'administration et les Directeurs financiers. Dans les faits, la responsabilité managériale se heurte à trois types d'obstacles ; politiques, structurels et réglementaires (Barilari, 2007). Les premiers renvoient à la question des statuts, celui des acteurs politiques et celui des acteurs administratifs, et des périmètres d'autorité et de compétences. La responsabilité managériale des RP en fait des acteurs de liaison entre des objectifs définis et contrôlés par l'autorité politique, ministre et Parlement, et des ressources régentées par l'autorité administrative et hiérarchique. Pour s'affirmer, leur autorité et le périmètre de leur compétence managériale impliquent donc 
qu'ils disposent d'un pouvoir discrétionnaire (Charreaux et Wirtz, 2006), qui ne remette pas en cause l'autorité politique, administrative ou hiérarchique.

Les seconds obstacles, d'ordre structurel, renvoient à la séparation qui caractérise le modèle français entre les directions de moyens, internes et externes à l'image des directions du ministère des finances, et les directions de missions que sont les directions en charge de la mise en œuvre concrète des politiques publiques. Cette séparation créé une tension car la logique des premières est celle du contrôle a priori des moyens, tandis que la logique des secondes est celle du contrôle a posteriori des résultats (Rochet, 2005). La question dépasse toutefois, les seules logiques d'action pour se poser en termes d'architecture et d'organisation de l'État. L'absence de révision des structures pour une plus grande délégation a en effet aboutit à la reproduction des mêmes logiques de fonctionnement. La question est d'autant importante qu'elle structure le dialogue entre le niveau central et le niveau déconcentré et détermine la pertinence des mécanismes d'allocation des moyens et le pouvoir des RP en la matière.

Les derniers obstacles sont d'ordre réglementaire et ont trait au système de contrôle, notamment financier, limitant les marges de manœuvre accordées aux acteurs de l'action publique. Ils cristallisent les deux autres catégories d'obstacles et posent la question de l'adaptabilité du modèle bureaucratique français dans l'ensemble de ses fondements (Duran, 2009). En effet, en basant la nouvelle architecture budgétaire sur la notion de programme qui fait correspondre l'unité de spécialité budgétaire, la politique publique et le périmètre d'exercice de la responsabilité managériale, la LOLF implique une révision du modèle d'exercice du pouvoir. Les programmes et les gestionnaires publics qui en sont responsables, ont ainsi été au centre de cette tension entre le pouvoir des politiques, celui des structures administratives et hiérarchiques et celui des structures managériales de la LOLF. Une tension qui se trouve aggravée par l'absence de mécanismes de coordination formels entre la responsabilité managériale et la responsabilité administrative.

En effet, l'absence de révision globale de l'organisation de l'État fait coexister deux structures, l'une formelle (structure hiérarchique) et l'autre informelle (structure LOLF). Or, seule le RP est appelé à rendre compte devant le ministre, le Parlement et autres assemblées, sur les résultats des actions publiques. Il assume donc la responsabilité des résultats sans disposer des marges de manœuvre administratives et/ou politiques nécessaires pour ce faire. La mise en œuvre d'une réelle dynamique d'évaluation, de responsabilisation et de reddition des comptes implique donc au moins deux conditions. La première est de rendre effective la responsabilité managériale attribuée aux responsables de programme, ce qui suppose comme le souligne Barilari (2007) de leur donner la légitimité et les marges nécessaires pour mener les relations de dialogue de gestion, de pilotage et d'évaluation, avec le ministre de rattachement, avec les échelons inférieurs mais également avec les autres directions de moyens internes ou externes. La seconde condition est de renforcer la responsabilité des politiques, ministres et Parlement, sur les missions, niveau supérieur aux programmes, et de leur permettre de disposer de dispositifs qui assurent une imbrication entre les performances individuelles et les performances des programmes.

La LOLF oblige donc à rompre avec une tradition française qui veut que la réforme de l'État soit distinguée de la réforme de la gestion publique (Greffe, 1999). En effet, la réforme des finances et des règles de la gestion budgétaire de l'État, semble difficile en l'absence d'une révision de l'organisation de l'État et des mécanismes de gouvernance. 
Aussi, la réforme met en évidence la nécessité d'aller au-delà de la dualité qui a longtemps caractérisé les structures publiques avec un modèle de régulation politico-administratif centralisé et des relations pyramidales hiérarchisées (Thoenig, 1987). En introduisant ce niveau intermédiaire, qu'est la régulation managériale, sans réellement remettre en cause le modèle existant, elle accentue donc de fait le flou entre les différents types de responsabilité.

Et de manière plus large, la réforme met en exergue l'équilibre difficile à trouver et l'effort d'adaptation nécessaire pour mettre en œuvre les doctrines du NPM (Hood, 2001 ; Osborne et Plastrik, 1998). En effet, si la doctrine du NPM prend les couleurs du paysage politique, structurel et économique qui l'accueille, ses principes intrinsèques impliquent des exigences spécifiques en termes d'évolutions organisationnelles et institutionnelles (Hood et Lodge, 2004). Aussi, comme pour d'autres réformes menées sous le label NPM, la LOLF est marquée par un enjeu d'équilibre : autonomiser et responsabiliser les administrations sans remettre en cause la légitimité politique de l'État.

Conformément au modèle wébérien en effet, la légitimité politique de l'État se fonde sur l'administration bureaucratique comme forme d'organisation rationnelle de l'action publique et sur la règle de droit comme instrument de l'action publique (Duran, 2009). Or, le NPM introduit une remise en question à deux niveaux (Duran, 2009). Au niveau de l'action publique en elle-même, qui n'est plus uniquement limitée aux activités des administrations. Il existe en effet, d'autres modes de mise en œuvre que la seule administration, ce qui produit une hybridation des modèles organisationnels car le pilotage de l'action publique dépasse le seul pilotage des administrations. Au niveau de la règle, le droit n'est plus le seul outil ou instrument de l'action publique, il doit cohabiter avec d'autres outils et plus particulièrement ceux du management. Par conséquent, s'il ne remet pas en cause la légitimité politique de l'État, le NPM interroge néanmoins son modèle d'exercice du pouvoir, ses structures, ses mécanismes de gouvernance et ses outils (Marchesnay, 2011).

\section{Conclusion}

Dans cet article nous avons traité du cas des responsables de programme, hauts fonctionnaires nommés par leurs ministres de rattachement pour assurer le pilotage de la mise en œuvre des politiques publiques et en rendre compte. Nous nous sommes intéressés plus spécifiquement à l'exercice de leur responsabilité managériale dans le cadre de la réforme LOLF et avons posé la question des marges de manœuvre dont ils disposent pour ce faire. L'analyse effectuée nous a permis de mettre en évidence les principaux facteurs limitatifs à l'exercice de la responsabilité managériale des responsables de programme : chevauchement des périmètres d'action, de compétences et d'autorité, insuffisance des marges d'action pour l'animation des échelons inférieurs, et lacunes en matière d'incitations à rendre compte et à faire l'objet de sanctions.

Nous avons montré ainsi qu'en introduisant cette dimension intermédiaire, qui est la dimension managériale, entre le politique et l'administratif sans réellement remettre en cause le modèle existant, la LOLF appelle une clarification des modes de partage des responsabilités et des dispositifs de reddition des comptes. Nous avons cherché ainsi à mettre en évidence le fait que les évolutions du cadre budgétaire et financier apportées par la LOLF demeurent incomplètes en l'absence d'une évolution du cadre institutionnel et organisationnel. En 
effet, à la différence d'autres réformes françaises telle que la rationalisation des choix budgétaires, la LOLF ne peut être limitée à des aménagements à la marge ou au simple usage d'un langage et d'outils gestionnaires. La doctrine NPM qu'elle véhicule et son essence systémique, imposent une révision de l'ensemble du cadre de l'action publique. Aussi, la réforme de la gestion publique est selon nous difficile en l'absence d'une réforme de l'État.

Notre analyse présente toutefois quelques limites qu'il convient de souligner. La principale limite est d'ordre méthodologique et concerne le matériau mobilisé. Nous nous sommes basés sur des données documentaires, plus particulièrement des rapports de la Cour des Comptes, qui présentent des limites en termes de pertinence mais également en termes de neutralité. Des données directes, sur la base d'entretiens avec les acteurs concernés, sont donc nécessaires pour enrichir et affiner l'analyse. En outre, ces données permettront d'approfondir les jeux et les enjeux politiques, sur les plans individuel et collectif, et d'explorer les compromis mis en œuvre par les acteurs au profit de l'action. 


\section{Rapports mobilisés pour l'enquête documentaire}

COUR DES COMPTES, (2011). La mise en œuvre de la loi organique relative aux lois de finances (LOLF). Un bilan pour de nouvelles perspectives. Rapport au Gouvernement - novembre 2011.

Arkwright E., De Boissieu C., Lorenzi JH. et Samson.J., (2007). Économie politique de la LOLF. La Documentation française, Paris.

Lambert A., Migaud D., (2005). La mise en œuvre de la loi organique relative aux lois de finances. Réussir la $L O L F$, clé d'une gestion publique responsable et efficace. Rapport au Gouvernement, septembre 2005.

ARTHUIS J., (2005). LOLF : Culte des indicateurs ou culture de la performance. Rapport d'information $n^{\circ} 220$ (2004-2005), fait au nom de la commission des finances, mars 2005.

\section{Bibliographie}

ALBERTINI J-B., (2000). Réforme administrative et réforme de l'État en France - Thèmes et variations de l'esprit de réforme de 1815 À nos jours. Economica, Paris.

BARTOLI A., (2009). Management dans les organisations publiques $3^{\mathrm{e}}$ Édition. Dunod, Paris

BARILARI A., (2007). La LOLF et la responsabilité des acteurs pour la mise en œuvre des politiques publiques In ARKWRIGHT E et al., L'économie politique de la LOLF, La Documentation Française, Paris.

BARILARI A. et BOUVIER M., La nouvelle gouvernance financière de l'État, LGDJ-Systèmes, 2004

BEVIR M., RHODES R.A.W. and WELLER P., (2003). Traditions of Governance: Interpreting the Changing Role of the Public Sector. Public Administration 81 (1), 1-17.

BEZES P., (2005). L'État et les savoirs managériaux : essor et développement de la gestion publique en France In LACASSE F. ET VERRIER PE. (dir) 30 ans de réforme de l'État. Expériences françaises et étrangères : stratégie et bilan, Édition Dunod, Paris.

CHARREAUX G. et WIRTZ P., (2006). Gouvernance des entreprises, nouvelles perspectives, Economica, Paris.

DE VISSCHER C. et VARONE F., (2004). La nouvelle gestion publique en action. Revue internationale de politique comparée 11 (2), 177-185.

DE VISSCHER C., (2004). Autorités politiques et haute administration : Une dichotomie repensée par la NGP? Revue internationale de politique comparée 2, 205-224.

DURAN P., (2009). Légitimité, droit et action publique. L'année sociologique 59 (2), 303-344.

DUSSART V. et ESPLUGAS P., (2000). L'ordonnance du 2 janvier 1959 : 40 ans après. Études de l'IRE DE, Toulouse.

EMERY Y. et GIAUQUES, (2005). Paradoxe de la gestion publique. L' Harmattan, Paris.

ERALY A. et HINDRIKS J., (2007). Le principe de responsabilité dans la gestion publique. Reflets et perspectives de la vie économique XLVI, p 193-208.

EYMERI JM., (2005). La gouverne au miroir du néo-management. Libres propos sur les gouvernants élus, les fonctionnaires gouvernants et l'activité gouvernante aujourd'hui », intervention de clôture du $14^{\text {e }}$ colloque international de la revue Politiques et Management Public, «Le management public à l'épreuve de la politique », IEP de Bordeaux, 17-18 mars 2005.

GREFFE X., (1999). Gestion publique. Dalloz, Paris.

HOCHEDEZ D., (2004). La formation de la loi organique du $1^{\text {er }}$ août 2001 : l'élaboration de la proposition de loi organique. Revue Française de Finances Publiques 86.

HOOD C. and LODGE M., (2004). Competency, Bureaucracy and Public Management Reform: a Comparative Analysis. Governance 17 (3), 313-333.

HOOD C., (2001). Relations entre ministres / politicies et fonctionnaires : l'ancien et le nouveau marchandage In PETERS B. et SAVOIE J. La gouvernance au XXI siècle : revitaliser la fonction publique, Les presses de l'Université Laval, Québec, 129-150. 
LE GALÈSE P., (2003). La réforme de l'État et la nouvelle gestion publique : mythe ou réalité. Revue Française d'Administration Publique 105-106 (1-2).

MATTEI P., (2007). Managerial and Political Accountability: the Widening Gap in the Organization of Welfare. International Review of Administrative Sciences 73 (3), 365-387.

MARCHESNAY M., (2011). Gouvernance et performance des organisations. Les limites de la doxa managériale. Innovations 36 (3), 131-145.

MAZOUZ B. et ROCHET C., (2005). De la gestion par résultats et de son institutionnalisation : quelques enseignements préliminaires tirés des expériences françaises et québécoises. Télescope 12 (3), 69-85.

MAZOUZ B. \& MATHAIS D., (2004). À nouvelle gouvernance, nouvelles compétences. Revue Internationale de Gestion 29 (3), 82-92.

MULGAN R., (2000). Accountability': An Ever-Expanding Concept? Public Administration 78 (3), 555-573.

OSBORNE D. and PLASTRIK P., (1998). Banishing Bureaucracy: The Five Strategies for Reinventing Government. Addison-Wesley, New York.

POLliTT C. and BOUCKAERT G., (2004). Public Management Reform. A Comparative Analysis $2^{\text {nd }}$ Ed. Oxford University Press, Oxford.

POLLITT C., (2002). Clarifying Convergence: Striking Similarities and Durable Differences in Public Management Reform. Public Management Review 4 (1), 471-492.

ROCHET C., (2005). Piloter l'évolution de l'État : Comment lier innovation dans les organisations et dans les institutions? Télescope 12 (3), 88-107.

THOENIG JC., (1987). L'ère des technocrates. L'Harmattan, Paris.

VERHOEST K., PETERS B.G., BOUCKAERT G. and VERSCHUERE B., (2004). The study of organizational autonomy: a conceptual review. Public Administration and Development 24 (2), 101-18.

WAINTROP F. et CHOL C., (2004). France : le pari d'une réforme systémique - Les enjeux de la réforme. Colloque annuel du GEAP, Ljubljan, 1-4 septembre 2004.

WACHEUX F., (1996). Méthodes Qualitatives et Recherche en Gestion. Economica, Paris. 